\title{
Diacronie
}

Studi di Storia Contemporanea

$\mathrm{N}^{\circ} 24,4 \mid 2015$

Le dittature militari: fisionomia ed eredità politica

\section{Le péronisme : du coup d'État militaire au justicialisme}

\section{Pascal Madonna}

\section{(C) OpenEdition \\ Journals}

\section{Édition électronique}

URL : http://journals.openedition.org/diacronie/3655

DOI : 10.4000/diacronie.3655

ISSN : 2038-0925

Éditeur

Association culturelle Diacronie

\section{Référence électronique}

Pascal Madonna, «Le péronisme : du coup d'État militaire au justicialisme », Diacronie [En ligne], №24, 4 | 2015, document 4, mis en ligne le 29 décembre 2015, consulté le 19 avril 2019. URL : http:// journals.openedition.org/diacronie/3655; DOI : 10.4000/diacronie.3655 


\title{
Diacronie
}

N. 24 | 4|2015 Le dittature militari: fisionomia ed eredità politica

4/

\section{Le péronisme: du coup d'Etat militaire au justicialisme}

\section{Pascal MADONNA*}

Par le putsch du 4 juin 1943, Juan Perón, alors simple colonel, se rapproche significativement du pouvoir. En s'appuyant sur une loge militaire, le GOU, il multiplie les postes au gouvernement sous la présidence du général Ramirez. En soutenant l'accession au pouvoir du général Farrel il gravit une autre marche et devient viceprésident. Sa pensée politique dépasse petit à petit le cadre militaire, la rencontre avec Evita précipite la rupture, emprisonné quelques jours, il est libéré sous la pression populaire et accède à la présidence en 1946. Comment Perón, membre éminent de l'armée et du GOU a-t-il pu débuter sa carrière politique en tant que participant à un coup d'Etat militaire pour en venir à être déposé par un autre environ 10 ans plus tard?

\author{
Dans l'Amérique du Sud, les Républiques \\ ne reposent que sur la puissance militaire, \\ toute leur histoire est une révolution \\ continue ${ }^{1}$.
}

(Friedrich Hegel)

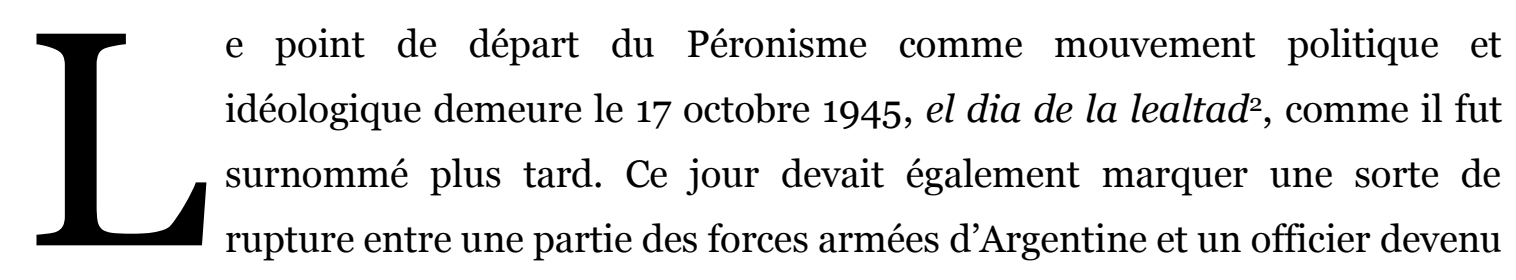

\footnotetext{
${ }^{1}$ HEGEL, Friedrich, Leçons sur la philosophie de l'histoire, Paris, Vrin, 1979, p. 70.

${ }^{2}$ Littéralement: «Le jour de la loyauté».
} 
trop influent auprès des masses populaires du pays, le colonel Juan Domingo Perón, futur général et président de la nation argentine. Dans l'histoire du XXe siècle, existe-til un homme qui fut plus que lui un sujet de controverse pour les historiens? Existe-t-il un homme politique qui ait autant influencé l'histoire de son pays avant et après sa mort? Le plus remarquable étant que cet officier de carrière ait réussi à s'affranchir des intérêts de la caste des officiers supérieurs dont il est issu et de la grande bourgeoisie au point de s'en faire détester et de perdre le pouvoir du fait d'un nouveau putsch. Comment Perón, membre éminent de l'armée et du Groupe des Officiers Unis a-t-il pu débuter sa carrière politique en tant que participant à un coup d'Etat militaire pour en venir à être déposé par un autre environ 10 ans plus tard? C'est un cheminement qui le dépassa toute sa vie. Longtemps après sa mort, le conflit qu'il avait entamé entre l'armée et tous ceux qui pouvaient se prévaloir de sa pensée, prit des proportions dramatiques et ce n'est qu'en 1983 que l'Etat argentin put enfin connaître la paix civile, avec la défaite cinglante du parti justicialiste aux élections libres.

\section{La place de l'armée en Argentine}

«La politique d'un Etat est dans sa géographie» aurait dit l'empereur Napoléon. En ce cas, un simple regard sur une carte de l'Argentine suffit à comprendre que le pays ne pouvait qu'être condamné à la division. A l'indépendance, l'absence d'un centre politique et économique unique provoqua d'inévitables divisions.

L'instabilité chronique de l'Argentine dans ses premiers pas en tant qu'entité étatique perdura durant ses 40 premières années d'existence. Les pulsions centralisatrices d'une partie de la population se heurtèrent assez vite à la volonté d'autonomie des caudillos et à leur souhait de voir émerger un Etat fédéral décentralisé. Les multiples guerres civiles entre les centralistes et les caudillos fédéralistes se soldèrent par la victoire finale de Bartolomé Mitre sur les fédéralistes en 1861 et son élection à la présidence en 1862. Assez ironiquement, ces multiples conflits n'avaient pas permis l'émergence d'un acteur indispensable à la stabilité étatique : une armée régulière3. D’un côté comme de l'autre, on était davantage confronté à des milices formées par les provinces et les grands propriétaires terriens. L'entrée de l'Argentine dans une ère de prospérité économique permit à l'armée régulière un développement rapide et radical, au point peut-être d'en faire l'acteur majeur de la politique nationale au XXe siècle.

3 ROUQUIE, Alain, Pouvoir militaire et société politique en république Argentine, Paris, Presse de la fondation nationale des sciences politiques, 1978, p.62. 


\subsection{Quels sont les facteurs de la puissance de l'armée dans la société argentine au début du XXe siècle ?}

C'est le successeur du président Bartolomé Mitre, Domingo Sarmiento, qui jette les bases de la professionnalisation de l'institution militaire après la création d'une armée nationale en 1864 en faisant voter en 1869 la loi de création du collège militaire de la nation, organe de formation des officiers, qui verra passer par ses portes de nombreuses futures hautes personnalités militaires, dont le général Juan Perón.

Les réformes qui touchent l'armée dans les années 1860-1870 sont le fruit des circonstances. En effet, de 1864 à 1870, la guerre de la triple alliance opposa le Brésil, l'Argentine et l'Uruguay au seul Paraguay. Bien loin d'une promenade militaire, le petit Paraguay en imposera durant 6 ans à ses trois voisins démographiquement bien plus puissants que lui. Au cours de la guerre, les présidents argentins Mitre et Sarmiento comprennent la nécessité de se doter d'un outil militaire puissant. A ce titre, ils vont s’inspirer des réformes établies par le maréchal Fransisco Solano López, dictateur du Paraguay. Tout comme lui, les deux chefs d'Etats vont adopter le système militaire prussien 4 .

La création en 1900 d'une école supérieure de guerre à Buenos Aires démontre la part dominante de l'influence allemande sur l'armée argentine. Son premier directeur, était un ancien officier prussien en retraite, tout comme la moitié des instructeurs qu'il amena avec lui durant les premières années d'existence de l'institution. Si l'on reçoit de nombreux officiers allemands dans les écoles militaires du pays, on envoie également beaucoup d'officiers argentins se former en Allemagne au début du XXe siècle. Avant la Première Guerre mondiale, entre 150 et 175 furent accueillis dans les institutions militaires impériales entre 1905 et 1914. Parmi ces officiers, on trouve ceux qui seront les chefs de l'armée à partir des années 1930.

C'est donc une armée de mieux en mieux organisée qui se développe au début du XXe siècle en Argentine. L'adoption de la conscription, en 19015, obligea également le monde militaire à évoluer sur d'autres points, comme sur son rôle d'intégration sociale de nouvelles élites. Jusque-là, l'armée était principalement entre les mains d'une oligarchie, souvent rurale. Or, les nécessités de la conscription, et peut-être l'influence allemande qui a très tôt promu ce comportement, poussèrent celle-ci à s'ouvrir à une

4 BONARDI, Laurent, «L'armée argentine et les relations Perón-Franco», in Revue historique des armées, 259, 2/2010, pp. 93-105.

5 ROUQUIE, Alain, Pouvoir militaire et société politique en république Argentine, cit. p. 71. 
nouvelle classe en cours d'émergence: la bourgeoisie, principalement citadine. Au tournant des années 40, le corps des officiers est principalement constitué de militaires issus de la bourgeoisie et des classes moyennes à une exception près: la Marine.

Un autre facteur de puissance réside dans l'influence de l'armée sur le secteur économique. ${ }^{6}$ Durant les années précédant la révolution de 1930, l’armée agit comme un moteur du processus d'industrialisation de l'économie du pays. Depuis le début du XXe siècle, le modèle économique argentin est celui de «l'agro-exportateur», puissant producteur de matières premières qu'il exporte partout dans le monde. L'Argentine importe les produits manufacturés dont elle a besoin, auprès des grandes puissances tels le Royaume-Uni ou les Etats-Unis d'Amérique. En 1922, sous la pression des militaires et plus particulièrement du général Mosconi et de ses collaborateurs, le gouvernement d'Yrigoyen prit des mesures pour faciliter la transition productive de l'économie et plus particulièrement, entreprit la création d'une entreprise pétrolière YPF (Yacimientos Petrolíferos Fiscales) - sous la forme d'un conglomérat allant de l'extraction à la commercialisation du pétrole7. Après le coup d'Etat de 1930, les milieux militaires plaident plus que jamais pour une industrialisation rapide du pays. Plusieurs tribunes publiées dans la "Revista Militar" par le capitaine Roberto Marambio et le colonel Manuel Savio, soulignent le rapport étroit entre l'industrie et la souveraineté nationale.

\subsection{La révolution de 1930: Point de départ de la «décennie infâme»}

Le 12 octobre 1928, le vieux «Caudillo» radical Hipólito Yrigoyen est élu pour la deuxième fois, avec plus de $57 \%$ des suffrages, à la présidence de la nation argentine, poste qu'il avait déjà occupé entre 1916 et 1922. Très vite, le monde militaire est choqué par les tentatives de réformes portées par les radicaux: introduction de critères politiques dans le système de promotion des gradés engendrant fatalement des injustices dans le tableau d'avancement et refus des demandes de financements formulées par les autorités militaires. L’idéologie germanique majoritaire dans la formation de l'armée permet également de mieux appréhender la conception de l'armée qui aurait un rôle politique à jouer dans les institutions.

Dès 1929, un accord transcende une petite partie de l'armée: il faut chasser Yrigoyen du pouvoir. Mais les modalités d'actions différent selon les chefs du parti

6 SUSANI, Bruno, Le péronisme de Perón à Kirchner une passion argentine, Paris, L'Harmattan, 2014, p. 27.

7 LARRA, Raúl, Mosconi: General del Petróleo, Buenos Aires, ED. Futuro, 1957, p. 166. 
militariste. La première présidence d'Yrigoyen avait déjà créé de nombreuses tentions avec les chefs de l'armée et on craint que la seconde ne soit pire encore. Deux projets de renversement concurrents voient le jour en 1930 : celui du général José F. Uriburu, privilégiant une action militaire forte et un changement radical du système politique et celui du général Agustín P. Justo, plus favorable à une union de tous les acteurs opposés aux radicaux, partis politiques inclus, afin d'obtenir la démission légale du caudillo, de nouvelles élections devant avoir lieu dans les semaines qui suivirent.

$\mathrm{Au}$ mois de septembre 1930, les conspirateurs du camp d'Uriburu se voient contraints, du fait du faible soutient des unités autour de la capitale, à se plier aux exigences du secteur légaliste ${ }^{8}$ de l'armée. Le 6 septembre, le coup d'Etat est en réalité plus civil que militaire: très peu d'officiers se joignent aux putschistes, ce qui n'empêche pas ces derniers de triompher. Yrigoyen est chassé du pouvoir; c'est le début de la décennie infâme, dans laquelle les militaires vont, avec l'aide des conservateurs, diriger le pays.

\section{Un colonel nommé Juan Domingo Perón}

De la jeunesse de Juan Domingo Perón, on sait relativement peu de choses 9 . Il est né le 8 octobre 1895 aux environs de Los Lobos. Il est le fils de Mario Tomás Perón et Juana Sosa, deux fermiers. Très tôt, il se destine à la carrière militaire et c'est en 1910 qu'il entre au collège militaire de la nation. Le 13 décembre 1913, le sous-lieutenant Perón est affecté au $12^{\mathrm{e}}$ régiment d'infanterie. Jusque-là, il n’y a pas grand-chose qui puisse le distinguer de tous les autres jeunes officiers de son temps, si ce n'est une origine sociale qui le place à peine dans la classe moyenne supérieure. Il entre en 1926 à l'école de guerre. Breveté d'état-major en 1929, il va orienter sa carrière autant sur le commandement effectif de terrain que sur l'étude de l'histoire militaire, ce qui le conduira à obtenir une chaire d'enseignant d'histoire militaire au collège militaire de la nation.

En septembre 1930, son soutient discret mais réel à la révolution le conduit au poste de secrétaire du ministère de la Guerre avant de recevoir une promotion au rang de major en décembre. Six ans plus tard, il obtient une nouvelle promotion au grade de lieutenant-colonel. Il est nommé attaché militaire à Santiago du Chili, poste dont il est

8 GONZALEZ ALEMAN, Marianne, «Le 6 septembre 1930 en Argentine: un coup d'Etat investi de révolutions», in Colloque: L'idée de Révolution en Amérique latine du 19e au $20 e$ siècle. Paris, 26-27 Janvier 2007, Paris, Nuevo Mundos Mundos Nuevos, 2007, URL:

$<$ https://nuevomundo.revues.org/5385 > [consulté le 12 septembre 2015].

9 ROLINAT, Jean-Claude, Qui suis-je? Juan Perón, Paris, éditions Pardès, 2013, p. 13. 
rappelé en 1938 pour prendre le commandement du $14^{\mathrm{e}}$ régiment d'infanterie de montagne au $1^{\mathrm{er}}$ janvier 1939. Il n'occupera ce commandement de terrain que quelques semaines avant de se voir désigné pour participer à une mission d'étude en Italie dans le but d'observer l'entraînement des troupes de montagne. De ce séjour, au-delà des observations militaires, Perón découvre les idées fascistes et plus particulièrement son vecteur social. A son retour au pays, il avance à nouveau dans la hiérarchie et obtient le grade de colonel. Il est promu attaché au centre d'instruction technique des troupes de montagne, dont il devient grand spécialiste. En mars 1942, il devient inspecteur des troupes de montagne à l'état-major de Buenos Aires avant de participer le 4 juin 1943, à la prise de pouvoir de l'armée qui mettra fin à la décennie infâme, de la même manière (bien que finalement beaucoup plus militaire) que celle-ci avait commencé: par un Pronunciamento.

\section{L'influence du GOU}

Le coup d'Etat de septembre 1930 inaugurait donc la période dite de la décennie infâme ${ }^{10}$ durant laquelle la démocratie argentine se délite peu à peu sous les coups des militaires. Cette caste militaire qui fait son entrée en politique est de plus en plus influencée par les milieux nationalistes. Le caractère troublé de cette époque participe à l'apparition de logias, qui peuvent se définir comme des groupements d'officiers à vocation politique. Le sigle GOU reçut plusieurs interprétations: Groupe pour l'Euvre d’Unification, Gouvernement-Ordre-Unité, mais la plus fréquemment retenue est celleci: Groupe des Officiers Unis ${ }^{11}$.

Si la logia de San Martín participe activement aux évènements de septembre 1930, le GOU n'apparaît officiellement qu'à partir de 1942. La création de cette loge militaire serait le fait de deux hommes, Juan Montes et Urbano de la Vega ${ }^{12}$, deux lieutenantscolonels, qui furent vite rejoints par plusieurs jeunes officiers supérieurs, dont Juan Domingo Perón. Du 6 septembre 1930 au 4 juin 1943, les gouvernements se succèdent, tous issus d'élections fortement entachées de fraudes et pratiquant une politique répressive envers les opposants politiques. C'est le retour des caudillos; le général Uriburu d'abord, remplacé en 1932 par le général Justo. A partir de 1938, le contrôle de l'armée sur le pouvoir politique argentin vacille lentement. Le successeur du général

${ }^{10}$ L'expression «Década Infame», que l'on a souvent traduit par «décennie infâme» ou «décade infâme», a été forgée par le journaliste péroniste José Luis Torres dans son ouvrage intitulé «La Década Infame» dans lequel il analysait la période.

${ }^{11}$ BONARDI, Laurent, «L'armée argentine et les relations Perón-Franco», cit., pp. 93-105.

12 GALASSO, Noberto, Peron formation, ascenso y caida, Buenos Aires, Colihué, 2006, p. 142. 
Justo est un civil : Roberto Marcelino Ortiz, mais celui-ci ne peut engager que de timides réformes avant d'être atteint par la maladie et de devoir laisser sa place à son vice-président: Ramón Castillo. C'est sous sa présidence que le GOU choisit de renverser l'Etat.

\subsection{Quelle est l'idéologie du GOU?}

Les membres du GOU ont forgé une idéologie proche de celles développées en Europe à cette période par des hommes comme Maurras en France, Ernst Von Salomon en Allemagne ou Ramiro de Maeztu ${ }^{13}$ et Primo de Rivera en Espagne. Cette pensée est axée sur plusieurs points:

Le caractère catholique, que l'on retrouve rapidement dans les premiers écrits revendicatifs des membres du GOU, Las bases de la Acción, puis quelques mois plus tard à nouveau dans Las nuevas bases. C'est sur la base de ces textes qu'est pris le décret du 31 décembre 1943 sous le gouvernement militaire de Pedro P. Ramirez. Le texte stipule: «L'école sans religion est une école antidémocratique et anticonstitutionnelle qui ne prépare pas l'enfant à l'honneur suprême auquel peut aspirer chaque Argentin, celui d'être président de la Nation». Au-delà de la sphère officielle, plusieurs membres du GOU affirment le caractère catholique de la révolution qu'ils souhaitent porter au sein des médias. Ainsi, un auteur anonyme en lien avec le GOU peut-il écrire dans le période catholique «El Pueblo»: «Le libéralisme laïc et agnostique est coupable d'avoir privé la Nation d'une doctrine morale». Une conséquence annexe de cette catholicité réaffirmée conduit le GOU, comme la plupart des milieux militaires, à condamner la franc-maçonnerie.

La deuxième caractéristique de l'idéologie du GOU correspond à la reconnaissance de la notion d'Hispanité. Par là même, les officiers dirigeant le GOU affirment l'appartenance à une identité culturelle hispanique, entamant un rapprochement avec l'Espagne du général Franco, et marquant leur désapprobation de l'influence anglaise durant les années suivant la fin de la Première Guerre mondiale sur l'économie argentine.

Enfin, la dernière caractéristique identifiable du GOU réside dans son militantisme nationaliste. Les membres du GOU ont forgé une idéologie nationaliste axée sur un double rejet : celui des forces socialistes inspirées par la doctrine marxiste et celui des partis conservateurs, jugés trop proches de l’impérialisme anglo-saxon.

13 FIGALLO, Beatriz, «Ramiro de Maetzu y la Argentina», in Res Gestae, 24, 2/1988, pp. 73-92. 


\subsection{La prise de pouvoir et l'arrivée de Perón}

Depuis le coup d'Etat de 1930, le monde militaire était divisé en deux blocs idéologiques: d'un côté les «libéraux» et de l'autre les «nationalistes». C'est donc à cette deuxième voie que se rattacheront les membres du GOU. Vers la fin de son mandat, Ramón Castillo avait pris le parti de soutenir comme successeur à la convention conservatrice la candidature de Robustiano Patron Costas. C'est le 4 juin 1943 que la convention devait se réunir, mais elle ne pourra jamais le faire: au petit matin, l'armée investit tous les points sensibles de Buenos Aires.

Castillo et ses ministres sont affectés à bord du Drummond: un dragueur de mines de l'Armada commandée par le vice-amiral Sabá Sueyro. Le général Arturo Rawson accède à la présidence pour... trois jours. Jugé trop favorable aux anglo-saxons, il est remplacé par le général Pedro Ramírez qui nomme le général Edelmiro Farrel à la tête du ministère de la Guerre. Le choix est essentiel ; le général Farrel, protecteur de Perón, est le futur successeur de Ramírez à la présidence. De plus, Perón peut désormais faire officiellement son entrée au gouvernement en tant que secrétaire d'Etat au Travail et à la Prévision. En dépit de son poste de secrétaire au ministère de la Guerre après septembre 1930, Juan Domingo Perón ne fera réellement son entrée en politique qu'en juin 1943. Dans le gouvernement Ramírez, tous les postes sont occupés par des militaires, à l'exception du ministère des Finances qui est l'attribut du seul civil: Jorge Santamarina. Le GOU ne souhaita pas organiser des élections dans la foulée du coup d'Etat de juin 1943, contrairement à ce que firent les militaires en septembre 1930, ce qui rendit l'action du gouvernement nécessairement plus complexe. Très vite, la Grande-Bretagne et les Etats-Unis d'Amérique qualifient le gouvernement de «fasciste». A l'intérieur, les relations sont tendues entre les différents acteurs politiques: la dissolution des partis politiques, la censure de la presse et le contrôle des gouvernements provinciaux n'empêchent nullement le pays de s'agiter.

A l’intérieur de la junte, les tensions se font jour entre les militaires libéraux, plus proches des milieux anglo-saxons, et les militaires nationalistes se présentant comme anti-impérialistes. Le général Ramirez renonce au pouvoir et la présidence échoue alors entre les mains du général Edelmiro Farrell, le 24 février 1944. Perón en profite pour gravir une autre marche du pouvoir. Huit jours après l'arrivée du général Farrell au pouvoir, son protégé est nommé ministre de la Guerre, position qu'il cumule avec le secrétariat d'Etat au Travail. Par cette promotion, Perón marque clairement son 
allégeance à la frange «nationaliste» de la junte militaire. Le 7 juillet 1944, Farrell le nomme même vice-président; Perón n'est plus qu'à une marche de la position suprême.

\section{Le point de rupture avec l'armée : Rencontre avec Eva Perón et naissance du péronisme}

De toutes les influences que le colonel, bientôt général, a connu ou connaîtra dans sa vie, aucune ne peut égaler celle qu'a exercée sur lui sa deuxième épouse: Eva Duarte, la fameuse Evita. Leur rencontre, lors d'une soirée de charité organisée à la suite d'un tremblement de terre à San Juan, en janvier 1944, va entraîner une succession d'événements qui vont marquer une rupture entre les militaires et le vice-président.

Tout de suite après cette soirée de gala commence une histoire d'amour, peut-être l'histoire d'amour la plus commentée du XXe siècle, entre l'actrice de 25 ans et un des hommes les plus puissants du pays, alors âgé de 49 ans. Immédiatement, cette relation est mal perçue par la bourgeoisie du pays et par la caste des officiers, particulièrement par les officiers supérieurs. Celle qu'on surnomme «la jument» n'a pas bonne réputation et n'appartient pas au même monde que la plupart des femmes d'officiers, d'autant que la passion de Perón pour Eva débouche parfois sur des interventions discrètes en sa faveur et c'est l'une d'elles qui faillit lui couter sa carrière.

A la demande de son amante, le ministre accepte de nommer Oscar Nicolini, protégé d'Eva, à la fonction de directeur des Postes. Or ce poste était déjà promis à un officier subalterne du général Eduardo Ávalos. Celui-ci, légitimement ulcéré par la décision de son ministre de tutelle, approche le général Farrell qui ne parvient pas à infléchir la décision de son subordonné. Finalement, dans la nuit du 8 au 9 octobre 1945, Avalos et quelques officiers supérieurs se mutinent contre le gouvernement révolution de palais en réalité - puisque Farrell n'est pas menacé. Il lui est seulement demandé de renvoyer Perón. ${ }^{14}$ En réalité cette anecdote, bien que révélatrice, ne suffit probablement pas à expliquer ce renvoi, en tant que membre du gouvernement Perón avait introduit plusieurs mesures favorables à la classe travailleuse dont l'amélioration des conditions de travail et la mise en place d'indemnités de licenciement ou d'accident de travail. Ces réformes, très populaires auprès des classes les plus pauvres, furent souvent mal considérées par des secteurs très influents de la société nationale. Ces derniers ne virent pas d'un mauvais œil l'éviction du ministre.

\footnotetext{
${ }^{14}$ Ce qu'il accepte assez facilement pour qu'aujourd'hui encore on ne puisse que s'interroger sur les réels sentiments du président envers son subordonné trop influent.
} 
Le 10 octobre, Perón, démissionnaire mais toujours en liberté, s'adresse à une foule de supporteurs devant l'immeuble du secrétariat au Travail. La foule acclame l'homme qu'elle a appris à aimer pour ses positions sociales depuis deux ans. Elle hurle «Perón président!» et ses cris parviennent aux oreilles effrayées des putschistes qui pensaient, déjà, en avoir fini avec lui. Deux jours plus tard, Perón est arrêté par le général Mittelbach avant d'être transféré au pénitencier de l'île de Martín García. Aucune troupe ne se rebelle contre l'emprisonnement du colonel ; la révolte viendra des civils, ceux qu'on surnommera les Descamisados. ${ }^{15}$ Dès les premières heures de la matinée du 17 octobre, une foule immense commence à se masser sur la Plaza de Mayo pour réclamer sa libération. Un peu avant minuit, le Líder apparaît au balcon présidentiel aux côtés du général Farrell. El día de la lealtad reste encore aujourd'hui une célébration péroniste.

Tout va désormais aller très vite pour le couple Perón. Afin de remercier Eva de son travail auprès des syndicats - travail ayant débouché sur les événements du 17 - Juan et elle se marient le 22 octobre. Le 14 novembre, le Partido Laborista, parti puissamment soutenu par les syndicats, est fondé. Il ne tarde pas à annoncer la candidature de Perón aux élections présidentielles programmées pour février 1946. Le 8 avril 1946, les résultats officiels tombent: c'est un triomphe pour Perón. Il est élu avec 1.500.000 voix contre 1.200.000 pour son concurrent. Pour entériner l'acceptation du résultat par l'armée, Perón est réintégré dans les forces armées le 31 mai 1946, avec le grade de général de brigade. Raccommodement provisoire, puisque la crise qui couve entre l'armée et le nouveau président n'en est qu'à ses débuts.

\subsection{Les relations de plus en plus tendues avec l'armée}

Perón est un militaire. A ce titre, il a majoritairement la confiance de l'armée à son arrivée au pouvoir. Peut-être aussi qu'une part non négligeable des militaires libéraux sont satisfaits de voir un militaire sortir victorieux des élections de 1946 - premières élections libres depuis le coup d'Etat de 1930 - mettant fin par là même à la décennie infâme. D'ailleurs, Perón comprend très vite qu'il ne pourra gouverner efficacement sans soutien de l'armée. Comprenant que ses principaux soutiens militaires sont issus de l'infanterie et non de la Marine, plus conservatrice que les autres corps d'armée, il fait choisir le ministre de la Guerre, les commandants en chef des forces armées et le

${ }_{15}$ SUSANI, Bruno, op. cit., p. 17. 
chef de la police fédérale parmi les membres du corps de l'infanterie. ${ }^{16}$ En corrélation avec sa politique, il entame également une réforme des écoles militaires permettant l'accès au corps des officiers à des membres des classes populaires, auprès desquelles le péronisme est particulièrement populaire. Malgré cela, les relations entre Perón et l'armée ne cessent de se dégrader. Les facteurs de cette dégradation sont multiples, mais on peut en retenir trois.

\subsection{L'éloignement de la doctrine du GOU}

Seul au pouvoir, le général Perón semble petit à petit renier un pan de l'idéologie du coup d'Etat du 4 juin 1943. Le principal reniement touche aux relations avec l'Eglise catholique qui sont de plus en plus difficiles. La majorité péroniste au Parlement, composée en partie de socialistes et de syndicalistes, n'est pas particulièrement favorable au décret de 1943, prévoyant l'enseignement religieux à l'école. En plus de ce problème de base, la hiérarchie ecclésiastique du pays se méfie du général, pour deux raisons: en premier lieu, il serait suspecté de vouloir "péroniser" l'Eglise ou d'assurer un contrôle un peu trop évident de la hiérarchie catholique. Ensuite, il oppose à un catholicisme bourgeois une approche plus sociale, souhaitant revenir à une religion plus proche des premiers siècles de la chrétienté: une religion pour les pauvres et les déshérités comme il le dit lui-même lors d'un discours dans le Chaco, le 10 avril 1948:

De même que tous ceux qui se disent démocrates ne le sont pas, tous ceux qui se disent catholiques ne s'inspirent pas de la doctrine chrétienne. Notre religion est une religion de pauvres, de ceux qui ressentent la soif et la faim de justice, des déshérités ${ }^{17}$.

Au-delà de tous ces éléments, la personne d'Evita fut là encore un élément déterminant dans la détérioration des relations entre son mari et l'Eglise catholique. Le plus surprenant étant qu'Eva était là encore une catholique très pratiquante et respectueuse de la hiérarchie ecclésiastique. Sa réputation, souvent très exagérée et paradoxalement, son engagement social qui fit ombrage au monopole qu'exerçait l'Eglise en cette matière depuis toujours en Amérique latine, créèrent une sorte de compétition malsaine. A ce titre, la création de la fondation Eva Perón fut un véritable

\footnotetext{
${ }^{16}$ BONARDI, Laurent, «L'armée argentine et les relations Perón-Franco», op. cit., pp. 93-105.

17 Discours du général Perón dans le Chaco, le 10 avril 1948 pour soutenir l'action sociale de l'évêque.
} 
camouflet contre les organismes de bienfaisance catholiques préexistants. Cette relation tendue fut particulièrement bien symbolisée par le voyage diplomatique entrepris au Vatican en 1947 par Eva Perón. Elle est reçue par le pape le 26 juin. Sa Sainteté le pape Pie XII ne lui accordera que dix minutes et... un chapelet.

\subsection{Le justicialisme : Une politique trop éloignée des intérêts de la bourgeoisie oligarchique dont sont toujours issus beaucoup d'officiers supérieurs}

Après sa victoire aux élections de 1946, Perón prend la décision d'appeler à la dissolution des partis qui l'ont soutenu durant la campagne et au rassemblement au sein d'un nouveau mouvement, le PURN (Partido Único de la Revolución Nacional). Le parti péroniste (nouveau nom en 1947) va très vite s'organiser autour de deux branches: une branche politique et une branche syndicale, puis trois avec la création du Parti péroniste féminin (1949). Ces trois branches se partageront par tiers les postes sur les listes électorales. Les définitions sur l'idéologie du mouvement péroniste sont nombreuses. Hélas, elles véhiculent souvent les clichés médiatiques habituels sur Juan Perón: fascisme, soutien aux nazis, démagogie et même dictature. La réalité est plus complexe, beaucoup plus complexe même. John William Cooke donne une définition succincte mais adéquate du péronisme: «le péronisme est le rassemblement des forces populaires articulé autour de la classe laborieuse».

Il existe plusieurs principes de base du justicialisme qui sont la souveraineté politique, la justice sociale, l’indépendance économique et la troisième position ${ }^{18}$. La première caractéristique du mouvement péroniste réside dans son ambition de trouver une troisième voie dans cette guerre froide naissante entre le camp des démocraties libérales et celui du monde communiste. Cette position, finalement assez proche de celle du général de Gaulle, n'empêcha pas l'ambassadeur américain, Spruille Braden, de financer toutes les activités anti-péronistes. Ce premier point fut particulièrement apprécié par les milieux militaires nationalistes qui rejetaient autant l'idéologie marxiste que l'influence américaine.

L'affirmation de la souveraineté nationale n'est pas non plus anodine. Elle est à mettre en relation avec la recherche d'une troisième voie, mais surtout avec le rejet des tentatives extérieures d’influencer la politique nationale. Cette volonté n'est en rien une formule politique traditionnelle; l'Argentine a vraiment du lutter contre les influences

${ }^{18}$ SUSANI, Bruno, op. cit., pp. 56-59. 
étrangères, parfois même lorsque l'appel à l'aide venait de l'intérieur du pays. En 1946, divers partis de droite comme de gauche souhaitaient une intervention des puissances alliées - particulièrement des Etats-Unis - pour contrer la candidature de Perón. ${ }^{19}$

Le souhait de développer une économie indépendante, en relation avec les deux points précédents, trouve son origine dans la fin du XIXe siècle et le début du XXe siècle, période où l'économie argentine fut exploitée durement par les banques anglaises ${ }^{20}$. Une des principales doctrines économiques du péronisme fut de développer une industrie forte, axée sur les ressources du pays, mais aussi de retrouver la direction des principaux services publics. Quant à la justice sociale, ce fut très probablement le point de division le plus net entre les péronistes et la haute bourgeoisie oligarchique, dont tout un secteur de l'armée était encore originaire. L'apparition d'une branche syndicale au sein du parti péroniste démontrait l'importance de la question sociale pour les cadres péronistes, ainsi qu'une volonté de ne pas être débordés par les partis marxistes. Cette justice sociale fut la clé du conflit qui opposa les péronistes aux classes dirigeantes.

\subsection{Eva Perón à la vice-présidence?}

A la fin de l'année 1951, les dignitaires du parti justicialiste poussèrent le couple Perón à former un ticket gagnant pour les prochaines élections présidentielles. En cas de victoire, Eva Perón deviendrait donc vice-présidente, la première femme à un poste aussi élevé en Amérique latine. Plus la probabilité de cette investiture augmentait, plus l'opposition se déchaînait, d'autant que la différence d'âge aurait pu impliquer une mort du général qui advienne plus tôt que celle de sa femme, laissant à celle-ci le poste de président.

Dans le cas où cette candidature aurait été officielle, il n'existait que peu de doutes sur la victoire finale du couple présidentiel. C'était plus que ne pouvait tolérer les militaires ; même les hauts gradés proches du mouvement péroniste ne voyaient pas cette candidature d'un bon œil. Le 22 août 1951, un grand rassemblement a lieu à Buenos Aires, au carrefour de l'avenue 9 de julio et de l'avenue Moreno. Environ un demi-million de personnes se presse au meeting dans l'espoir d'entendre

19 La révélation des câbles Wikileaks a permis d'apprendre que lors de la présidence de Nestor Kirchner, certaines factions à l'intérieur du pays en appelèrent à des gouvernements étrangers pour renverser le chef péroniste ou en tant cas l'affaiblir.

${ }_{20}$ Sur ce sujet nous pouvons conseiller l'excellent ouvrage de: DIAZ, Alejandro, CARLOS, Federico, Essays on the economic history of the Argentine Republic, New Haven, Yale University Press, 1970. 
l'officialisation de la candidature conjointe des Perón. Pourtant, le couple sait qu'il n'en est déjà plus question. Avant la conférence, l'armée s'est faite entendre par la voix du général Franklin Lucero, ministre de la Guerre. Celui-ci a clairement fait comprendre à Perón que les forces armées désapprouvaient le projet. S’il passait outre, les officiers passeraient à l'action ${ }^{21}$. Le 31 août, Evita annonce officiellement qu'elle ne sera pas candidate aux côtés de son mari. Ce désistement n'empêche pas une première tentative de coup d'Etat militaire, le 28 septembre 1951, menée par le général Benjamín Menéndez.

Le coup est encore trop mal préparé et l'aura de Perón auprès des militaires est encore vivace. Bien renseigné, Perón prend contact avec des troupes sures ainsi qu'avec les dirigeants de la CGT. Très vite, l'état de guerre interne est proclamé. Quant aux syndicats, ils proclament la grève générale dans tout le pays. Rapidement, la situation rentre dans l'ordre. Le soulèvement manquait cruellement d'effectifs. Le coup de semonce est néanmoins lancé: le divorce entre le péronisme et l'armée ne tardera plus à être prononcé. Après sa réélection à la présidence aux élections du 11 novembre 1951, le général doit faire face à de nouveaux défis, particulièrement économiques mais aussi sociaux et sociétaux, les relations avec l'Eglise catholique ne cessant de se dégrader. Perón craint toujours une action violente de l'armée qui accepte de moins en moins la «péronnisation» de sa hiérarchie.

\section{Le renversement de Perón: un coup d'état militaire oui, mais de la part d'une petite partie de l'armée?}

Au tournant de la deuxième élection présidentielle remportée par le général Perón, celui-ci doit affronter un véritable front anti-péroniste dont les principaux acteurs furent les partis d'opposition, les grands propriétaires mais surtout l'Eglise et l'armée. Pourtant, le peuple continue à se mobiliser massivement pour apporter son soutien au général. Lors des élections générales du 11 novembre 1951, les deux tiers des votants apportent leurs suffrages au parti justicialiste. La mort d'Eva Perón le 16 juillet 1952 n'apaise pas les détracteurs qui y voient plutôt un signe d'affaiblissement du péronisme.

${ }^{21}$ FRASER, Nicholas, NAVARRO, Marysa, Evita the real life of Eva Peron, New-York, W.W. Norton and Company, 1996, p. 116. 


\subsection{Le bombardement de la Place de Mai}

Un deuxième soulèvement militaire a lieu le jeudi 16 juin 1955. Bien que raté, il ouvre la voie à celui qui, trois mois plus tard, emportera une première fois le régime péroniste. Bien qu'avortée, cette tentative rentrera dans l'histoire comme le bombardement de la place de Mai. Ce jour-là, 34 avions de l'aéronavale bombardent la Place de Mai, faisant au total entre 364 et 405 morts selon les sources. Au même moment sur terre, un bataillon des fusiliers marins essaya, en vain, de prendre d'assaut la Casa Rosada. Les personnels des 34 avions ayant fait couler tant de sang trouvèrent refuge en Uruguay et les fusiliers-marins durent se rendre. La modicité des troupes engagées ne doit pas cacher la réalité du divorce entre une partie des forces armées et le président. En réalité, le général Menéndez a frappé seul alors que deux autres complots se formaient déjà autour du colonel Suárez et du général Lonardi. Le vieux cavalier qu'était Menéndez, ne supportant plus l'attente avant les élections présidentielles, a préféré prendre les devants, seul ou presque.

\subsection{Le renversement du régime péroniste par une fraction de l'armée}

La grande répétition ayant eu lieu trois mois auparavant, un nouveau coup d'Etat éclate le 16 septembre 1955. A minuit, les forces navales se soulèvent à Rio Santiago sous les ordres de l'amiral Rojas. Rétrospectivement, il est possible de constater via ce soulèvement qui chassa du pouvoir le général Perón pour 18 ans, que l'armée est demeurée majoritairement fidèle au Líder argentin. Dès les premières heures de l'insurrection, celle-ci va d'échec en échec. L'infanterie de Marine échoue à prendre la ville de Rio Santiago, les rebelles ne disposent d'aucune aviation, et l'artillerie du régiment Azul, loyal au gouvernement, pilonne la base navale. Le 17, dans la soirée, l'amiral Rojas est contraint d'ordonner l'évacuation totale des effectifs.

$\mathrm{Au}$ même moment, la base navale de Puerto Belgrano se soulève également. Par son éloignement du centre politique du pays, elle bénéficie de certaines facilités. Là encore, la Marine joue un rôle de premier plan. Devant l'avancée de la révolte, quelques régiments d'infanterie et d'artillerie se rallient. Autour de la capitale, aucune garnison ne se rebelle. Le général Aramburu échoue à son tour à soulever la garnison de blindés de Curuzú-Cuatiá, principal troupe blindée du pays, qui choisit la loyauté à l'Etat. En réalité, au matin du 18 septembre, la rébellion n'a pas embrasé le pays comme l'espéraient les généraux rebelles. Le collège militaire lui-même, dont la valeur symbolique reste importante, demeure fidèle au régime. 
Le premier foyer rebelle de Rio Santiago est défait. La capitale ainsi que la majorité des troupes d'infanterie et d'aviation - souvent des sous-officiers dont le corps reste particulièrement fidèle au péronisme - affichent leur loyalisme. Il n'en demeure pas moins que la rébellion dispose de deux poches défensives dans le pays: Puerto Belgrano ainsi que les provinces de San Luis et de Mendoza, sous les ordres du général Lagos et de sa puissante $2^{\mathrm{e}}$ armée. La ville de Córdoba, alors entre les mains des rebelles, devient l'un des centres névralgiques symboliques du coup d'Etat. Les troupes loyalistes encerclent la ville entre le 17 et le 18 ; l'assaut final semble imminent et laisse peu de chances de résistance.

Militairement, la rébellion n'a qu'une seule carte en main : la Marine, qui s'est jointe massivement au coup d'Etat. Celle-ci adopte une nouvelle stratégie à partir du 19 : la destruction d'objectifs économiques névralgiques. Dès le matin du 19, la Marine bombarde et détruit de nombreuses raffineries le long de la côte atlantique. Au moment de s'en prendre à la raffinerie de La Plata, une nouvelle très importante parvient au commandement rebelle: le général Perón a remis ses pouvoirs au commandement militaire. Le général Franklin Lucero annonce un cessez-le-feu et l'ouverture de négociations avec les rebelles. Par un message à la nation, le Líder explique sa décision: «Si mon tempérament de lutteur me pousse à combattre, mon patriotisme et mon amour pour le peuple m’invitent au renoncement personnel $»^{22}$. Perón, dans ce dernier message à la nation, réaffirme sa croyance en l'armée, seule garante de l'unité de la nation. Il reste un militaire jusqu'au bout, peut-être persuadé que la majorité de l'armée lui étant acquise, son départ ne saurait être définitif. Il espère probablement créer ainsi une nouvelle alliance au sein du péronisme entre les syndicats officiels et les troupes loyalistes contre une fraction de l'armée. Peut-être espère-t-il également que cette alliance le conduise à obtenir des moyens politiques plus importants.

Mais Perón se trompe. L'édifice péroniste se fissure largement durant les quelques jours du coup d'Etat. La Confédération Générale du Travail lâche «Le premier travailleur». Le comité directeur confédéral, organe directeur du syndicat, prend ses distances dans les jours qui suivent et lance des appels au calme. Les hauts fonctionnaires, souvent membres du parti justicialiste, préfèrent assurer leurs places dans la société post-péroniste que risquer leurs vies pour un Líder dont la démission résonne comme un aveu de faiblesse. Devant ses multiples défections, il ne reste à Perón qu'un seul espoir: l'armée loyaliste. Or celle-ci se trouve face à un choix difficile: accepter la lutte fratricide contre d'autres militaires, et donc de nouvelles pertes au sein

22 ROLINAT, Jean-Claude, op. cit., p. 84. 
de l'armée comme de la société civile, ou bien trouver une solution politique qui conviendrait aux rebelles.

Le choix est vite fait: le 20 septembre a lieu une entrevue au sein du croiseur rebelle General Belgrano. La junte militaire qui remplace Perón accepte les conditions de la Marine: la révolution est victorieuse. Le général Eduardo Lonardi est nommé «chef de la révolution» à la tête d'un gouvernement révolutionnaire provisoire. Ce putsch semble si faible militairement qu'on ne peut que s'étonner d'une fin si misérable du régime. Peu d'unités, hormis dans la Marine, ne se soulèvent. Même au sein des officiers supérieurs, le nombre de factieux est faible. Les sous-officiers, massivement péronistes, refusent de participer au soulèvement. Ils sont soutenus en cela par les officiers subalternes. L'infanterie, à l'exception de l'infanterie de Marine, est presque exclusivement fidèle au général et à l'Etat péroniste.

\subsection{Quelles étaient les motivations des putschistes?}

Elles étaient celles qui avaient déjà été affirmées maintes fois par les fronts antipéronistes ; à savoir le retour à une situation économique, politique et sociale qui était celle existant en Argentine avant l'arrivée au pouvoir du Líder. Plusieurs réformes prises après le coup d'état nous renseignent sur les motivations du nouveau régime, retenons particulièrement la décision de fermer tous les établissements sociaux crées par la fondation Eva Perón ${ }^{23}$. La répression s'abat sur tous les cadres, réels ou supposés, du parti péroniste, environ 50.000 prisonniers politiques sont arrêtés.

Mais une partie non négligeable de l'armée reste fidèle au président en fuite, la preuve étant qu'un nouveau soulèvement pro-péroniste a lieu le 7 juin 1956, des groupes de militaires et de civils sous les ordres du général Valle tentent de soulever plusieurs régiments dans différentes régions. Le soulèvement est rapidement vaincu et plusieurs centaines de militants sont arrêtés, quelques militaires sont fusillés mais l’ordre est rétabli.

\section{De 1955 à 1973: lutte mortelle entre l'armée et les péronistes}

Très tôt après la chute du général un vaste mouvement de résistance prend corps entre une partie de la société civile, fidèle au péronisme et l'armée, petit à petit purgé de

\footnotetext{
23 La nouvelle junte militaire ferma notamment les foyers pour orphelins de la fondation Eva Perón et licencia les assistantes sociales qui y travaillaient pour les remplacer par des agences pour l'emploi.
} 
ses éléments trop proche du général déchu. Le général Aramburu a dissous le Parti justicialiste par décret le 5 mars 1956 mais la branche syndicale du parti péroniste n'est pas morte, son inertie durant le coup d'Etat est contrebalancée par une grande activité quelques mois plus tard. L'armée tenta bien de briser le pouvoir des syndicats liés au péronisme par des décisions très durs: arrestation des dirigeants syndicaux, exils et condamnation à la prison et même l'utilisation du parti communiste pout tenter de contrebalancer l'adhésion des masses populaires au programme social du justicialisme. Par leurs capacités, les syndicats, initialement une des branches du péronisme, deviennent la colonne vertébrale de résistance du mouvement. Parallèlement au combat syndical des groupes d'action clandestins se mobilisent à travers tout le pays, principalement composés de civils, dans le but d'entreprendre des actions de sabotage contre des infrastructures militaires et économiques ou encore simplement pour se réunir et commenter les dernières directives du président en exil. Le développement de ces groupes fut de plus en plus important au point qu'en 1959 un groupe armé prit le maquis et adopta le nom d' "Armée de libération nationale-Mouvement péroniste de libération».

En quelques mois les soutiens militaires du péronisme semblèrent complètement s'étioler, l'armée faisait front commun contre un adversaire: Perón. Bien qu'il faille reconnaître que des tendances plus conciliante envers le parti proscrit ont pu exister face à l'opposition agressive contre tout accord que la marine tenait pour règle. De 1958 à 1966, les militaires, sous couvert d'un gouvernement civil issu des élections, assurèrent eux-mêmes la réalité du pouvoir, de plus en plus impopulaire l'armée doit faire face à une situation économique peu favorable ainsi qu'à une opposition forte de la société civile, le vote blanc, consigne péroniste, arrive en tête à de nombreuses élections.

En 1966 un nouveau coup d'Etat militaire, mené par le général Juan Carlos Onganía, semble apaiser un temps le pays. Néanmoins l'incapacité des militaires à réformer une situation qui dure depuis presque dix ans paralyse toute action gouvernementale sur le long terme. Paradoxalement cette situation renforça les péronistes, qui purent jouir à partir des années 60 de la dynamique créée par un vaste mouvement de politisation de la jeunesse, partout dans le monde (mai 68 en France par exemple) et donc également en Argentine, cette jeunesse qui s'identifia facilement à un mouvement proscrit, mené par un chef légendaire en exil et refusant la vassalité au bloc soviétique ou américain.

Des groupes de guérilla se réclamant du vieux Lider se forment à travers tout le pays à la fin des années 60 et au début des années 70, les Forces Armées Péronistes 
(FAP), les Forces Armées Révolutionnaires (FAR) ou encore les Descamisados montent des opérations d'assassinats ou de sabotages ${ }^{24}$ ainsi que les Montoneros qui parvinrent à devenir une organisation très puissante vers 1973. En octobre 1970 un nouveau Conseil supérieur du mouvement justicialiste est nommé, cet organe est le reflet de la volonté de Perón d'unir les différentes tendances du mouvement mais aussi de négocier avec la junte militaire présidé par le général Lanusse. Celui-ci fait voter une nouvelle loi organique en juillet 1971 prévoyant une période de transition d'une année aboutissant à de nouvelles élections générales, libres, pour mars 1973. Ne pouvant se présenter lui-même Perón désigna Héctor Cámpora, vieux militant, pour le représenter, celui-ci l'emporta assez facilement et accéda, pour peu de temps, à la présidence, mettant fin par la même à la dictature militaire. Le 17 novembre 1972, le célèbre exilé pouvait débarquer à l'aéroport d'Ezeiza, il était de retour au pays après dix sept ans loin de sa patrie. Il sera reélu magistralement à la présidence en septembre 1973 et, paradoxe remarquable, il choisira sa troisième femme, Isabel Martínez de Perón, comme vice-présidente alors même que le choix d'Evita avait précipité l'opposition militaire. Ce choix sera déterminant car le $1^{\text {er }}$ juillet 1974, le vieux Lider, âgé de 78 ans, succombait à un nouvel infarctus.

\section{Conclusion}

A la mort du général c'est bien sûr son vice-président qui doit prendre sa place, en l'occurrence il s'agit de la troisième femme du grand homme, Isabel. L'armée, déjà fortement ébranlée par le retour au pouvoir de son mari, ne tarde guère à agir contre Isabelita. Le 24 mars 1976, celle-ci est kidnappée en plein vol par des militaires qui l'expédient en captivité dans les Andes, où elle restera durant cinq années. C'est ainsi que la dernière dictature militaire s’installa en Argentine, la dernière mais aussi la plus dure, l'objectif est clair, finir d'abattre le parti justicialiste ainsi que tous les souvenirs liés au péronisme. Les militaires déclenchèrent une répression sans aucune mesure avec ce qu'elle avait pu être entre 1955 et 1973 en vue de détruire toutes les représentations syndicales, sociales ou politiques qui leur étaient hostiles. De 1976 à la chute définitive du régime militaire en 1983 les argentins subirent ce qu'on appela «le terrorisme d'Etat», système caractérisé par l'utilisation illégale de l'appareil étatique à des fins répressives. Les résultats de cette répression sont encore étudiés aujourd'hui mais on estime que le nombre total de disparus se situent aux environs de 30 ooo

24 DABENE, Olivier, L'Amérique latine à l'époque contemporaine, Paris, Édition Armand Collin, 2011, p. 147. 
personnes, le nombre de mort est aux alentours de 10 ooo personnes pour environ 750.000 emprisonnés ${ }^{25}$.

La pitoyable issue de la guerre des Malouines en 1982 a sonné le glas de la junte militaire. Une nouvelle fois les forces armées devaient renoncer à l'exercice du pouvoir politique; les prétoriens doivent rendre les armes. Néanmoins le parti péroniste, épuisé, perd les élections générales de 1983. Il ne lui suffit plus d'évoquer le souvenir presque mythique du général et d'Evita pour l'emporter ${ }^{26}$. Le mouvement péroniste et le parti justicialiste en particulier doivent se renouveler pour recevoir de nouveau les suffrages de l'Argentine. Cette "rénovation", plus libérale, emportée par des personnalités telles Carlos Menem aboutirent à des désaccords profonds entre les différents courants du péronisme mais rendirent les relations péronisme-armée plus apaisées. L'échec de Duhalde, candidat officiel du parti, aux élections de 1999, sera vite rattrapé, puisque son adversaire victorieux, Fernando de la Rua, doit démissionner deux ans plus tard victime de la fronde populaire, le congrès lui désigna comme remplaçant... Eduardo Duhalde. Au début des années 2000 c'est à nouveau un couple qui prend en main la destiné du parti justicialiste et de l'Argentine, Nestor et Cristina Kirchner vont l'un après l'autre exercés la fonction de président de la nation argentine, le couple renouvelle la doctrine sociale de la pensée péroniste en mettant en place des programmes de grands travaux et de nationalisations.

\footnotetext{
25 SUSANI, Bruno, op. cit., p. 131.

${ }^{26}$ ROUQUIE, Alain, «L’Argentine après les militaires», in Politique étrangère, 49, 1/1984, pp. 113-125.
} 


\section{${ }^{*}$ L'auteur}

Pascal Madonna est diplômé de l'institut d'études politiques d'Aix-en-Provence, spécialité «Histoire militaire, géostratégie, défense et sécurité» ainsi que de l'université de droit AixMarseille en spécialité «Droit international, droit européen et droit des étrangers». Auteur d'un mémoire sur les sociétés militaires privées en mer ; il est actuellement doctorant en histoire contemporaine, sa thèse s'intitule «Les volontaires français dans les guerres de Yougoslavie, 1991-1995». Il a reçu en 2015 une bourse du ministère de la défense français.

URL: < http://www.studistorici.com/progett/autori/\#Madonna >

\section{Per citare questo articolo:}

MADONNA, Pascal, «Le péronisme: du coup d'Etat militaire au justicialisme», Diacronie. Studi di Storia Contemporanea : Le dittature militari: fisionomia ed eredità politica, 29/12/2015,

URL:< http://www.studistorici.com/2015/12/29/madonna_numero_24/ >

\section{Diacronie Studi di Storia Contemporanea $\widehat{\beta}$ www.diacronie.it}

Risorsa digitale indipendente a carattere storiografico. Uscita trimestrale. redazione.diacronie@hotmail.it

Comitato di redazione: Jacopo Bassi - Luca Bufarale - Elisa Grandi - Antonio César Moreno Cantano - Deborah Paci - Fausto Pietrancosta - Alessandro Salvador - Matteo Tomasoni - Luca Zuccolo

Diritti: gli articoli di Diacronie. Studi di Storia Contemporanea sono pubblicati sotto licenza Creative Commons 3.0. Possono essere riprodotti e modificati a patto di indicare eventuali modifiche dei contenuti, di riconoscere la paternità dell'opera e di condividerla allo stesso modo. La citazione di estratti è comunque sempre autorizzata, nei limiti previsti dalla legge. 